\title{
Estimation of unsteady aerodynamic forces using pointwise velocity data
}

\author{
F. Gómez ${ }^{1} \dagger$, A. S. Sharma ${ }^{2}$ and H. M. Blackburn ${ }^{1}$ \\ ${ }^{1}$ Dept. of Mechanical and Aerospace Engineering, Monash University, VIC 3800, Australia \\ ${ }^{2}$ Aerodynamics and Flight Mechanics, \\ University of Southampton, Southampton SO17 1BJ, UK
}

(Received ?; revised ?; accepted ?. - To be entered by editorial office)

A novel method to estimate unsteady aerodynamic force coefficients from pointwise velocity measurements is presented. The methodology is based on a resolvent-based reducedorder model which requires the mean flow to obtain physical flow structures and pointwise measurement to calibrate their amplitudes. A computationally-affordable time-stepping methodology to obtain resolvent modes in non-trivial flow domains is introduced and compared to previous existing matrix-free and matrix-forming strategies. The technique is applied to the unsteady flow around an inclined square cylinder at low Reynolds number. The potential of the methodology is demonstrated through good agreement between the fluctuating pressure distribution on the cylinder and the temporal evolution of the unsteady lift and drag coefficients predicted by the model and those computed by direct numerical simulation.

Key words: resolvent analysis, time-mean flows, unsteady aerodynamic coefficients

\section{Introduction}

Unsteady motions in fluid mechanics, owing to unsteady separations and vortex shedding, lead to unsteady aerodynamic loads of concern in multiple engineering applications, such as flight mechanics, wind engineering, acoustics and dynamic aeroelasticity. The identification of unsteady aerodynamic coefficients is especially critical if new air vehicle configurations are tested or if the flight envelope is extended beyond traditional manoeuvres (Brunton, Rowley \& Williams 2013). Unsteady aerodynamic models are derived either from wind tunnel testing or directly from flight test data because unsteady simulations of realistic configurations are likely to remain unaffordable (Spalart et al. 1997). Besides classical force balance instrumentation, non-intrusive strategies to estimate unsteady aerodynamic forces from particle image velocimetry (PIV) are also well-known (Kurtulus, Scarano \& David 2007; van Oudheusden 2013). These methods are based on combining experimental data with the governing equations in such a way that, provided with time-resolved velocity fields, a surface or volume integration of the Navier-Stokes equations can yield the pressure field, and hence the unsteady pressure forces. A limitation of the methodology is that three-dimensional time-resolved PIV is required to obtain three-dimensional velocity fields, and hence recover corresponding pressure fields.

In the present work we employ a methodology to estimate unsteady aerodynamic forces that is able to overcome the need for time-resolved three-dimensional velocity fields. Similarly to PIV-based approaches, the present methodology is also based on the combination of measurements with the Navier-Stokes equations. However, instead of employing threedimensional time-resolved snapshots of the velocity, the inputs of the methodology are

$\dagger$ Email address for correspondence: francisco.gomez-carrasco@monash.edu 


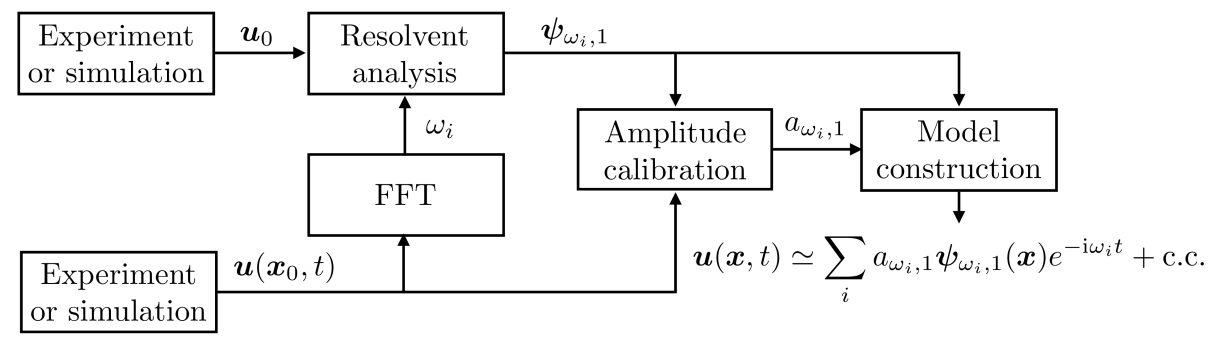

Figure 1. Diagram of the construction of the model. The mean flow and pointwise measurement inputs are on the leftmost blocks. A fast Fourier transform (FFT) of the probe signal provides the active frequencies $\omega_{i}$ to be explored in the resolvent analysis of the mean flow. The dominant resolvent modes $\psi_{\omega_{i}, 1}$ corresponding to the active frequencies are calibrated with the probe signal to obtain the amplitudes coefficients $a_{\omega_{i}, 1}$. A linear combination of the weighted resolvent modes provides an approximation of the fluctuating velocity and pressure. The fluctuating pressure is included in the fluctuating velocity vector $\boldsymbol{u}=(u, v, w, p)^{\mathrm{T}}$ for convenience.

the time mean flow and point measurements of the velocity. (We note that the mean flow field can notionally also be obtained from point measurements.) The use of the mean flow is motivated by the resolvent decomposition of McKeon \& Sharma (2010). A Reynolds decomposition applied to the Navier-Stokes equations reveals that the unsteady motions are dominated by the properties of a resolvent operator depending on the mean flow and spatial derivatives. This resolvent operator acts a forcing-to-response transfer function at each temporal frequency, hence the mean flow restricts the possible unsteady motions that may exist in the flow. A singular value decomposition (SVD) of the resolvent operator typically reveals that, at each particular frequency, there is a dominant unsteady flow structure with amplification ratio greater than other possible motions.

The feasibility of employing these dominant motions as a basis for the creation of reduced-order models of the fluctuating velocity field was recently demonstrated by Gómez et al. (2016) for flow in a rectangular lid-driven cavity. The present work expands on that theme to include the fluctuating pressure field, hence allowing estimation of fluctuating forces on an immersed body, and in addition employs a novel matrix-free time-stepping algorithm to estimate resolvent SVD modes, allowing them to be readily calculated in non-trivial flow domains. As for the method of Gómez et al., amplitudes of resolvent modes used in the reduced-order model are calibrated using pointwise measurements of the velocity. The new methodology is applied to the estimation of fluctuating forces imposed by the flow around an inclined square cylinder.

\section{Description of the methodology}

Figure 1 illustrates schematically the construction of the model employed to estimate the unsteady forces. The time mean flow $\boldsymbol{u}_{0}(\boldsymbol{x})$ and a pointwise measurement $\boldsymbol{u}\left(\boldsymbol{x}_{0}, t\right)$ of the velocity history are the inputs, corresponding to the leftmost blocks. In principle, mean flow and probe information could be obtained independently either from experiments or simulations. A spectral analysis of the probe signal $\boldsymbol{u}\left(\boldsymbol{x}_{0}, t\right)$ identifies the active frequencies $\omega_{i}$ to be explored in the resolvent analysis of the mean flow. The dominant resolvent modes $\psi_{\omega_{i}, 1}$ arising from the resolvent analysis corresponding to the active frequencies are calibrated with the probe signal to obtain the amplitudes coefficients $a_{\omega_{i}, 1}$. A linear combination of the weighted resolvent modes then provides an approximation of the fluctuating velocity and associated pressure fields. 


\subsection{Resolvent decomposition}

We follow a similar derivation of the resolvent decomposition as that proposed by Luhar et al. (2014). This derivation differs from that by Gómez et al. (2016) in that the pressure is explicitly taken into account, instead of projecting the velocity onto a divergence-free basis. A Reynolds decomposition is applied to the total velocity $\hat{\boldsymbol{u}}(\boldsymbol{x}, t)=\boldsymbol{u}_{0}(\boldsymbol{x})+\boldsymbol{u}(\boldsymbol{x}, t)$, with $\boldsymbol{u}(\boldsymbol{x}, t)$ being the fluctuating velocity which may be decomposed as a sum of temporal Fourier modes

$$
\boldsymbol{u}(\boldsymbol{x}, t)=\sum_{i} \boldsymbol{u}_{\omega_{i}}(\boldsymbol{x}) \mathrm{e}^{-\mathrm{i} \omega_{i} t}+\text { c.c. }
$$

The flow is assumed to be statistically steady thus the frequencies $\omega_{i}$ are real. A similar decomposition may be applied to the nonlinear terms, leading to $\boldsymbol{f}_{\omega_{i}}=-(\boldsymbol{u} \cdot \boldsymbol{\nabla} \boldsymbol{u})_{\omega_{i}}$. These decompositions lead to a formulation of the Navier-Stokes equations as

$$
\begin{aligned}
\boldsymbol{u}_{0} \cdot \boldsymbol{\nabla} \boldsymbol{u}_{0} & =-\nabla p+R e^{-1} \nabla^{2} \boldsymbol{u}_{0}+\boldsymbol{f}_{0} \\
\boldsymbol{u}_{\omega_{i}} & =\mathcal{H}_{\omega_{i}} \boldsymbol{f}_{\omega_{i}},
\end{aligned}
$$

with $\mathcal{H}_{\omega_{i}}$ being the resolvent operator of the Navier-Stokes for each frequency $\omega_{i}$. The mean flow equation 2.2 corresponds to $\omega=0$ and Reynolds stress $\boldsymbol{f}_{0}$ denotes the interaction of the fluctuating velocity with the mean. The fluctuating pressure augments the fluctuating velocity vector as $\boldsymbol{u}=(u, v, w, p)^{\mathrm{T}}$ so the resolvent operator imposes the continuity equation

$$
\mathcal{H}_{\omega}=\left(-\mathrm{i} \omega\left[\begin{array}{ll}
\mathcal{I} & 0 \\
0 & 0
\end{array}\right]-\left[\begin{array}{cc}
\mathcal{L} & -\boldsymbol{\nabla} \\
\boldsymbol{\nabla}^{\mathrm{T}} & 0
\end{array}\right]\right)^{-1}\left[\begin{array}{ll}
\mathcal{I} & 0 \\
0 & 0
\end{array}\right]
$$

with $\mathcal{L}$ being the Jacobian of the Navier-Stokes equations and $\mathcal{I}$ an identity matrix. This operator represents how the fluctuating velocity $\boldsymbol{u}_{\omega}$ is driven by nonlinearity $\boldsymbol{f}_{\omega}$ in Fourier space, hence it is useful to inspect its amplification properties via a singular value decomposition (SVD)

$$
\mathcal{H}_{\omega}=\sum_{m} \boldsymbol{\psi}_{\omega, m} \sigma_{\omega, m} \boldsymbol{\phi}_{\omega, m}^{*}
$$

where $\boldsymbol{\psi}_{\omega, m}$ and $\phi_{\omega, m}$ are two orthonormal basis termed response and forcing modes respectively. The superscript $*$ indicates conjugate transpose and the subscript $m$ indicates the ordering of the modes, ranked by the amplification rate given by the corresponding singular value $\sigma_{\omega, m}$ under the $L_{2}$ energy norm. The key of the resolvent decomposition is the projection on the nonlinearity onto the forcing modes (McKeon \& Sharma 2010), hence the fluctuating velocity can be written as a linear combination of response modes

$$
\boldsymbol{u}_{\omega}=\sum_{m} \boldsymbol{\psi}_{\omega, m} \sigma_{\omega, m} \chi_{\omega, m}
$$

where the unknown scalar coefficients $\chi_{\omega, m}$ are the projection of nonlinearity onto forcing modes and represent the forcing driving the velocity fluctuations (Gómez et al. 2016).

Equation 2.6 is an exact representation of the Navier-Stokes equation because no assumption other than a statistically steady flow has been used. On the other hand, it is useful to exploit the values taken by the amplification $\sigma_{\omega, m}$ in order to construct a reduced-order model of the fluctuating velocity. In presence of a single dominant flow feature such as a centrifugal instability (Gómez et al. 2016) or a critical layer response (McKeon \& Sharma 2010), the first singular value $\sigma_{\omega, 1}$ is usually much larger than the second one $\sigma_{\omega, 2}$, hence, at a particular frequency $\omega_{i}$, irrespective of the values taken by $\chi_{\omega, m}$, it can be assumed that the projection of nonlinearity onto the first response $\boldsymbol{\psi}_{\omega, 1}$ is 
much larger than onto the rest. As such, the low-rank properties of the resolvent operator can be employed to yield a rank-1 model

$$
\boldsymbol{u}_{\omega} \simeq \boldsymbol{\psi}_{\omega, 1} a_{\omega, 1}
$$

where the amplitude coefficients $a_{\omega, 1}=\sigma_{\omega, 1} \chi_{\omega, 1}$ represent the amount of nonlinearity being amplified. Under this rank-1 assumption, the fluctuating velocity (and pressure) can be expressed as

$$
\boldsymbol{u}(\boldsymbol{x}, t) \simeq \sum_{\omega} a_{\omega, 1} \boldsymbol{\psi}_{\omega, 1}(\boldsymbol{x}) \mathrm{e}^{-\mathrm{i} \omega t}+\text { c.c. }
$$

hence this assumption provides a convenient model in which the velocity fluctuations at each frequency are parallel to the first singular response mode corresponding to that frequency. This rank-1 assumption has proven to be adequate in previous investigations of pipe, channel and cavity flows (McKeon \& Sharma 2010, Moarref et al. 2013; Gómez et al. 2016).

\subsection{Amplitude calibration}

Obtaining resolvent modes $\boldsymbol{\psi}_{\omega, 1}$ can be computationally challenging in complex three-dimensional geometries even using time-stepping methods. However, only a small number of modes corresponding to the relevant or active frequencies in the flow are computed in practice. In the absence of further information, the active frequencies of the flow are identified via a Fourier analysis of a pointwise measurement of the flow. As highlighted in figure 1 the probe information can be obtained independently of the mean flow. Here we provide an extension of the calibration method developed by Gómez et al. (2016) to obtain the unknown amplitude coefficients that close the model (2.8) by using directly the same pointwise measurements of the velocity that have been previously employed for the identification of the active frequencies. At a particular spatial location $\boldsymbol{x}_{0}$, the reduced order model of the fluctuating velocity satisfies

$$
\boldsymbol{u}\left(\mathbf{x}_{0}, t\right) \simeq \sum_{i=1}^{N_{\omega}} \boldsymbol{\psi}_{\omega_{i}, 1}\left(\boldsymbol{x}_{0}\right) a_{\omega_{i}, 1} \mathrm{e}^{-\mathrm{i} \omega_{i} t}+\text { c.c. } .
$$

with $N_{\omega}$ representing the number of active frequencies (or discretized frequency bins) of the flow. Although each scalar component of 2.9 contains $N_{\omega}$ unknowns, it can be evaluated at a number of different time instants $N_{t}>N_{\omega}$, such that the solution is amenable to a least-squares approximation. We note that the spatial structure of the fluctuating velocity is restricted by the response modes, hence the pointwise calibration of the amplitude coefficients serves to capture the temporal behaviour of the fluctuating velocity. The solution of 2.9 in a least-squares sense is given by

$$
\mathcal{A}=\boldsymbol{\Psi}^{+} \mathcal{U}\left(\boldsymbol{x}_{\mathbf{0}}, t\right)
$$

with the $3 N_{t} \times N_{\omega}$ matrix $\boldsymbol{\Psi}$ containing the values of the three velocity components of the resolvent modes and their complex conjugates at the spatial location $\mathbf{x}_{0}$ at $N_{t}$ different times, the $N_{\omega} \times 1$ vector $\mathcal{A}$ representing the unknown amplitude coefficients, and the $3 N_{t} \times 1$ vector $\mathcal{U}$ contains the values of the velocity at the spatial location $\boldsymbol{x}_{0}$ at different times. The superscript + denotes pseudo-inverse. The dimensions of the least-squares problem 2.10 are much smaller than that of the SVD computations and its solution is straightforward. 


\section{Time-stepping strategies for resolvent analysis}

Although the simplest way to obtain numerically the resolvent modes is to assemble the resolvent operator and perform a SVD, this is difficult in practice owing to the massive computational requirements resulting from the large dimensionality of the operator associated to flows with two or three non-homogeneous spatial directions. Thus, iterative methods are preferred.

The main idea of iterative methods is that the singular values of the resolvent operator are the eigenvalues of $\mathcal{H}_{\omega} \mathcal{H}^{*}{ }_{\omega}$, and the response and forcing singular vectors correspond to the eigenvectors of $\mathcal{H}_{\omega} \mathcal{H}^{*}{ }_{\omega}$ and $\mathcal{H}^{*}{ }_{\omega} \mathcal{H}_{\omega}$ respectively. As such, the action of the resolvent operator and its conjugate transpose on a forcing vector could enable a matrix-free iterative power method to obtain the SVD of $\mathcal{H}_{\omega}$.

Following the rank-1 hypothesis posed in 2.1 , only the first singular vectors at each active frequency are required for the construction of the reduced-order model in 2.8 . In this context, Monokrousos et al. (2010) and Lu \& Papadakis (2014) showed that obtaining the dominant singular vectors of the resolvent is equivalent to finding the optimal harmonic forcing of the forced linearized Navier-Stokes equations

$$
\partial_{t} \boldsymbol{u}(\boldsymbol{x}, t)=\mathcal{L} \boldsymbol{u}(\boldsymbol{x}, t)+\boldsymbol{f}_{\omega}(\boldsymbol{x}) \mathrm{e}^{-\mathrm{i} \omega t} .
$$

The long time integration of (3.1) lead to the harmonic relation (2.3), if all transient effects vanish. Consequently, the optimal forcing and response vectors of $\mathcal{H}_{\omega}$ can be obtained from an optimization problem in a time-stepping context. A Lagrange function for the optimal forcing in (3.1) can be constructed as

$$
\begin{aligned}
\boldsymbol{L}\left(\boldsymbol{u}_{\omega}, \boldsymbol{f}_{\omega}, \boldsymbol{v}_{\omega}, \sigma^{2}\right) & = \\
& \left(\boldsymbol{u}_{\omega}, \boldsymbol{u}_{\omega}\right)-\left(\boldsymbol{v}_{\omega},\left(-\mathrm{i} \omega \boldsymbol{I}-\mathcal{L}^{*}\right)^{-1} \boldsymbol{u}_{\omega}-\boldsymbol{f}_{\omega}\right)-\sigma_{\omega}^{2}\left(\left(\boldsymbol{f}_{\omega}, \boldsymbol{f}_{\omega}\right)-1\right),
\end{aligned}
$$

where the objective function is the fluctuation energy represented by the energy norm $\left(\boldsymbol{u}_{\omega}, \boldsymbol{u}_{\omega}\right)$. The first Lagrange multiplier $\boldsymbol{v}_{\omega}$ enforces that the response and forcing satisfy the forced linearized Navier-Stokes equations (3.1) and the second Lagrange multiplier $\sigma_{\omega}^{2}$ enforces a unit energy norm to the optimal forcing vector. Variations of $\boldsymbol{L}$ with respect to the fluctuating velocity $\boldsymbol{u}_{\omega}$ and to the forcing $\boldsymbol{f}_{\omega}$ yield respectively

$$
\begin{aligned}
& \boldsymbol{v}_{\omega}=\left(-\mathrm{i} \omega \mathcal{I}-\mathcal{L}^{*}\right)^{-1} \boldsymbol{u}_{\omega}, \\
& \boldsymbol{f}_{\omega}=\boldsymbol{v}_{\omega} / \sigma_{\omega}^{2}
\end{aligned}
$$

The combination of the outcome of the optimization problem $(3.3)$ and $(3.4)$ with the (2.3) leads to the eigenvalue problem

$$
\boldsymbol{\psi}_{\omega, 1}=\sigma_{\omega, 1}^{-2}(-\mathrm{i} \omega \mathcal{I}-\mathcal{L})^{-1}\left(\mathrm{i} \omega \mathcal{I}-\mathcal{L}^{*}\right)^{-1} \boldsymbol{\psi}_{\omega, 1}
$$

where the fact that the optimal forcing solution corresponds to the first (most amplified) forcing mode $\boldsymbol{\psi}_{\omega, 1}$ has been exploited. An iterative matrix-free power method can be then applied to the eigenvalue problem (3.5) in order to obtain the dominant eigenvalue. However, as described by Monokrousos et al. (2010), time-stepping does not directly provide a solution of $(2.3)$ or (3.3) and the forced linearized and adjoint equations need to be integrated long enough such that the transient dynamics vanish and the response is harmonic. Note that the long integration of the adjoint of (3.1)

$$
\partial_{t} \boldsymbol{v}(\boldsymbol{x}, t)=\mathcal{L}^{*} \boldsymbol{u}(\boldsymbol{x}, t)+\boldsymbol{f}_{\omega}(\boldsymbol{x}) \mathrm{e}^{\mathrm{i} \omega t},
$$

provides a harmonic solution for 3.3 .

Although the algorithm originally proposed by Monokrousos et al. (2010) is successful in the sense that it is matrix-free, a number of difficulties arise in practice. The time 


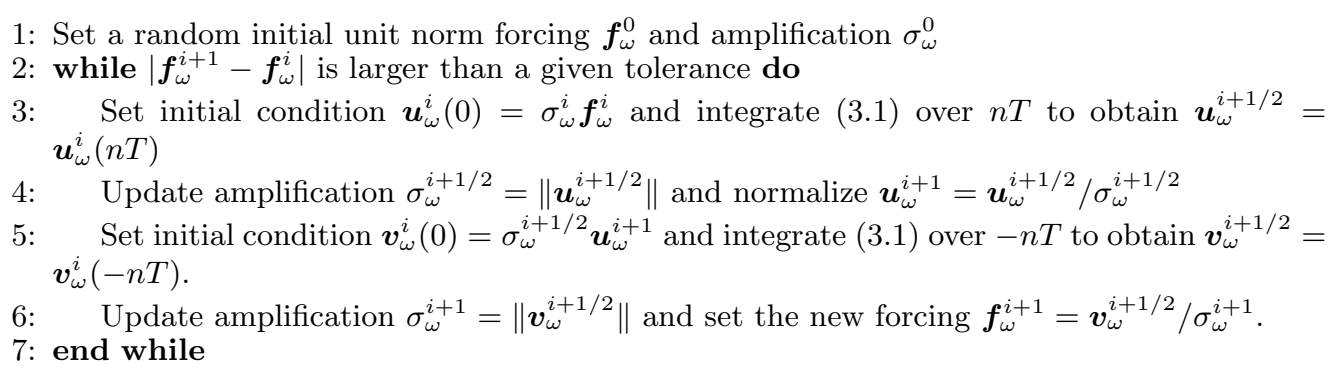

TABLE 1. Modification of the algorithm proposed by Monokrousos et al. (2010).

integration required of (3.1) until a harmonic response is obtained can be very long, hence the method may not be computationally affordable. The method is particularly slow at low frequencies. Also, the complex velocity response $\boldsymbol{u}_{\omega}$ is obtained via a Fourier transform of $\boldsymbol{u}(t)$ during one period, hence it could have a different phase at each iteration step. Finally, the response of (3.1) can be susceptible to large transient growths depending on the initial condition. Even if $\boldsymbol{u}_{\omega}$ and $\boldsymbol{f}_{\omega}$ are close to convergence, the transient can be significant if the two vectors do not have their relative phase fixed by the resolvent.

A modification of the time-stepping approach of Monokrousos et al. (2010) which is able to cope with the above-mentioned limitations is proposed by allowing the velocity field to take on complex values into the forced direct and adjoint linearized Navier-Stokes equations. The use of a complex variable has the advantage that is it is not necessary to perform a Fourier transform of the response during one period to obtain $\boldsymbol{u}_{\omega}$. Once the response of (3.1) is harmonic, any snapshot of the complex vector $\boldsymbol{u}^{\prime}(\boldsymbol{x}, t)$ represents its Fourier transform $\boldsymbol{u}_{\omega}$ at some particular phase. Furthermore, if the time integration is taken as an integer number $n$ of periods $T=2 \pi / \omega$ the response and force vectors obtained remain with the same relative phase imposed by the resolvent during a complete iteration. This feature avoids possible sources of transient effects during iterations.

In addition, the integration time can be limited to a small number of periods $n$ such that the strongest transients are vanished but the flow is not yet exactly periodic. This permits obtaining an estimation of the amplification value and of the response and forcing vector in relatively short integrals. The estimated $\sigma$ is then employed between each direct and adjoint iteration to generate a initial condition for the forced equations (3.1). This initial condition can be understood as a preconditioner, and it approaches the correct response as the estimated value $\sigma$ gets closer to the exact value. As a result, the transient effects are damped and the harmonic response is achieved after a few iterations.

All these features permit obtaining the most amplified modes using a smaller computational effort than with the original algorithm of Monokrousos et al. (2010). The modified algorithm is listed in table 1. In practice, the harmonic response is also assessed by monitoring the fluctuation energy. If the difference between the maximum and minimum fluctuation energy is less than a given tolerance within one period, the response is considered harmonic and the integration stops. Thus each iteration may require a number of integration periods less than $n$.

\subsection{Validation and comparison of the method}

A validation of the computation of the resolvent modes via time-stepping is carried out against results obtained using an in-house matrix-forming shift-invert method. The chosen validation case is the flow enclosed in a square lid-driven cavity at $R e=1200$ 

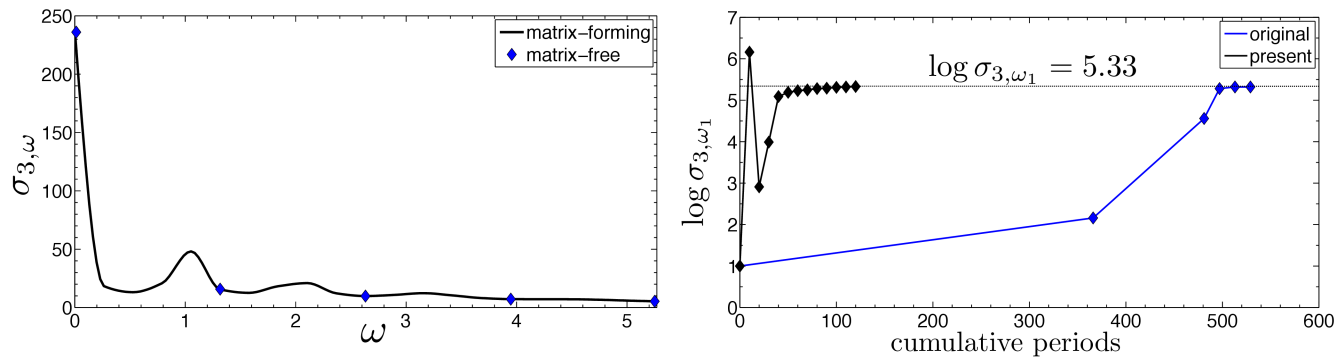

FigURE 2. (left) Validation of the method: amplitude of the first resolvent modes in frequency at $\beta=3$ obtained with the matrix-forming and time-stepping matrix-free methods. (right) Comparison of the original time-stepping methods envisaged by Monokrousos et al. (2010) and the present approach using $n=5$ : cumulative integration periods until convergence of the amplification. Diamond symbols denote the estimated value at each iteration. $\beta=3, \omega_{1}=5.26$. The tolerance error is set to 0.005 and the two methods start with the same initial condition.

with a periodic span $\Lambda=0.945$ (Gómez et al. 2016). A good agreement between the two methods is observed in figure 2 (a). However, for the problem examined, while the matrix-forming approach requires the storage and evaluation of a $\mathcal{O}\left(N^{2}\right)$ matrix, the time-stepping approach only needs two planes with $\mathcal{O}(N)$ degrees of freedom to deliver the same results. In the case presented, this evaluates to $3 \times 64^{2}$ against $\left(3 \times 64^{2}\right)^{2}$.

The optimal value of the parameter $n$ depends on the problem and, for the present case, we have observed that the method typically converges in a few iterations using $n=5$. In other words, five periods are enough to damp the most significant transient effects in the present case. Figure 2(b) shows a comparison of the present method with the original time-stepping methods envisaged by Monokrousos et al. (2010). We observe that although the proposed methods requires a larger number of iterations, it needs significantly less integration periods to achieve convergence. In this example, the number of the required integration periods until convergence goes from 540 periods to 116 periods, which translates into a $\sim 80 \%$ saving of $\mathrm{CPU}$ time.

\section{Application to the flow around an inclined square cylinder}

The flow past a two-dimensional inclined square cylinder may serve as a model for the flow around a non-trivial bluff body and it is a good compromise between computational affordability and complex flow features (Sohankar et al. 1998, Yoon et al. 2010). Beyond the critical Reynolds number, the wake becomes unsteady presenting asymmetric vortex shedding, thus this flow is interesting for the investigation of unsteady lift and drag forces.

Although the present methodology seems more appealing for experimental works, the mean flow and pointwise measure inputs to the model have been obtained via direct numerical simulation (DNS) using a spectral-element solver (Blackburn \& Sherwin|2004). A rectangular computational domain defined in $[-16,20] \times[-14,14]$ has been discretized with 236 spectral elements. The square cylinder has a unit side length and its centroid is located at $(x, y)=(0,0)$. Temporal and spatial convergence has been achieved with a polynomial expansion of order 11 in each element and using a second-order temporal scheme with $\Delta t=8.5 \times 10^{-3}$. A constant velocity $(u, v)=(\cos \alpha, \sin \alpha)$ is imposed at the inlet of the domain, no-slip boundary condition at the cylinder wall and Neumann boundary conditions are imposed at the outlet. The Reynolds number based on the cylinder side length $D$ and the modulus of the inlet velocity is fixed to $R e=100$. The angle of attack is set to $\alpha=10^{\circ}$.

The inputs to the model corresponding to the leftmost block in figure 1 obtained via 

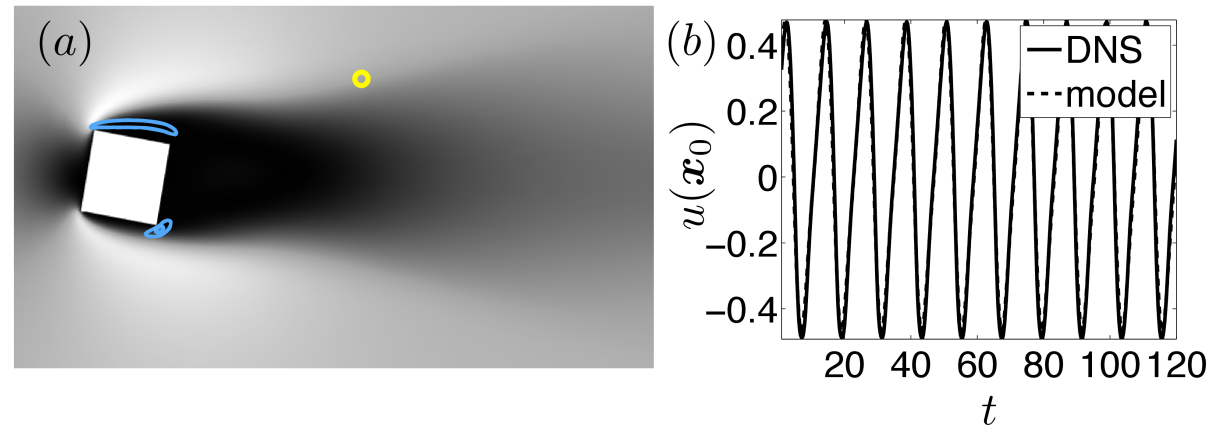

FIgURE 3. Inputs to the model corresponding to the leftmost block in figure 1 (a) Streamwise velocity contours of the mean flow, colored from black to white. Blue contour lines indicate $40 \%$ and $80 \%$ of the maximum of the kinetic energy corresponding to $\phi_{\omega_{1}, 1}$.(b) Evolution of the fluctuating streamwise velocity in the wake at the location $\boldsymbol{x}_{0}=(3,1.5)$, measured from the DNS and fitted to the model. The probe position is highlighted in (a) with a yellow circle.

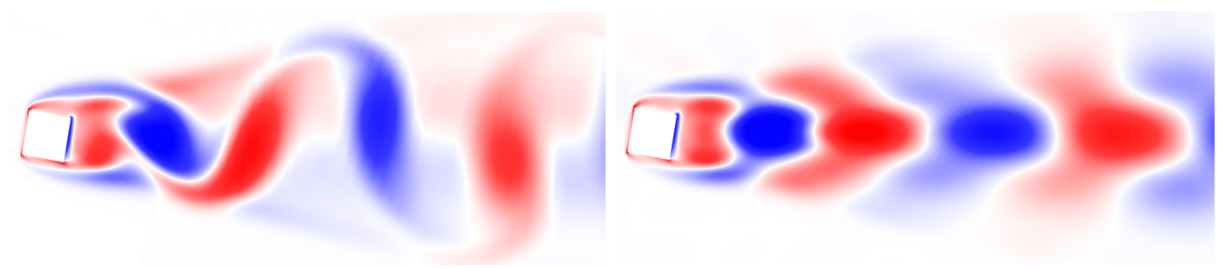

FIGURE 4. Comparison of the vorticity fields obtained from (left) DNS and (right) resolvent-based model via calibration of the amplitude against the probe data in figure 3(b). Colored contours represent $\pm 1 / 3$ of the maximum and minimum vorticity

DNS, mean flow and a single pointwise measurement of the velocity, are shown in figure 3 . The probe is located at a random point in the wake of the cylinder where the shear is non-zero and it serves to identify a single dominant frequency $\omega_{1}=0.908$, hence only one resolvent mode corresponding to that frequency needs to be computed. The dynamics of the self-interaction of this mode in this kind of flows could be relevant (Noack et al. 2003 ) thus it may be convenient to also consider the first harmonic $\omega_{2}=2 \omega_{1}$.

The iterative time-stepping algorithm described in $\S 3$ has been employed to obtain the two resolvent modes required to construct the reduced-order model. The forced linearized Navier-Stokes equations and their adjoint version have been solved with the same spectral-element solver employed for DNS. The boundary conditions for each equations are described by Barkley et al. (2008), however two set of boundary conditions at the inlet have been tested the forced adjoint equations, (i) an extended domain $[-40,20] \times[-14,14]$ with Dirichlet boundary conditions and (ii) the same domain employed for the DNS with a non-physical forcing $-m(\boldsymbol{x}) \boldsymbol{u}(\boldsymbol{x}, t)$ applied at $-16<x<12$ to force a zero-amplitude of the forcing mode at $x=-16$. Both boundary conditions have provided similar results, thus the former has been adopted on account of smaller computational requirements.

A comparison of the vorticity fields obtained from DNS and the resolvent-based model via calibration of the amplitude against the probe data in figure $3(\mathrm{~b})$ is shown is figure 4. It is remarkable that flow in the region around the cylinder, and hence the unsteady separation, is accurately predicted by the model, despite the probe being located approximately three side lengths from the cylinder. On the other hand, the structure of the wake far from the cylinder present obvious discrepancies. Although the general feature of vortex shedding is also reproduced, the DNS presents additional features that the present resolvent-based mode does not capture, like a consecutive and opposite vertical displace- 

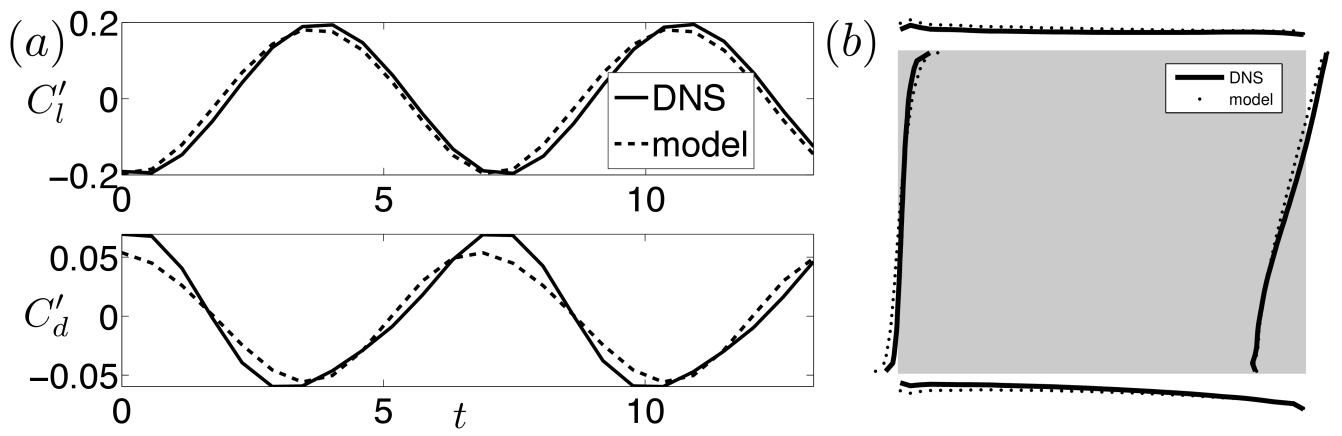

Figure 5. (a) Comparison of the temporal evolution of the unsteady lift and drag coefficients calculated via DNS and predicted by the present methodology. (b) Representation of the fluctuating pressure distribution along the sides of the cylinder at a random instant calculated via DNS and predicted by the present methodology. Each cylinder side acts as a $x$-axis while their normal direction indicates the relative value of unsteady pressure at that location.

ment of the vortex cores. An inspection of the spatial structure of the forcing mode that drives the resolvent modes, shown in blue isolines in figure 3(a), reveals that their maxima are located within the boundary layer of the cylinder, thus the discrepancies between the model and DNS can be attributed to this observation. As such, we presume that the additional dynamics of the far wake are governed by additional subdominant resolvent modes associated with the shear in the wake not considered in the present model.

The present approach is validated by measuring the unsteady lift and drag coefficients defined as:

$$
C_{l}^{\prime}=\int_{\delta \Omega_{c}} p(\boldsymbol{x}) \boldsymbol{n} \cdot \boldsymbol{e}_{y}, \quad C_{d}^{\prime}=\int_{\delta \Omega_{c}} p(\boldsymbol{x}) \boldsymbol{n} \cdot \boldsymbol{e}_{x} .
$$

where $\delta \Omega_{c}$ denotes the boundary of the cylinder, $\boldsymbol{n}$ is the normal vector around the square cylinder while $\boldsymbol{e}_{x}$ and $\boldsymbol{e}_{y}$ are the unit vectors in the streamwise and normal direction, respectively. At the present value of $R e$, the contributions of the viscous forces are negligible. Nevertheless, they could be taken into account using the present methodology. A good agreement between the temporal evolution of the unsteady lift and drag coefficients calculated via DNS and predicted by the present methodology is shown in figure 5(a). This results is consistent with the accurate prediction by the model of the near field around the cylinder shown in figure 4 . To provide further insight, the fluctuating pressure distribution along the sides of the cylinder at a random instant is represented in figure 5(b). The resemblance between the pressure distributions corresponding to DNS and the resolvent-based model supports the good agreement between the temporal evolution of the unsteady lift and drag forces. Finally, the same approach has been carried out using different pointwise measurements. As remarked by Gómez et al. (2016), similar results have been obtained provided that the probe is always positioned at a location where the fluctuating velocity is significant. However, this is not an issue because the locations where the fluctuating velocity is significant can be inferred from the spatial structure of the resolvent modes.

\section{Conclusions}

A novel method to estimate unsteady aerodynamic coefficients via pointwise measurements has been presented. The methodology requires two inputs (i) the mean flow and (ii) temporal information from a probe. In principle, both inputs could be obtained either simultaneously or independently. Although we believe the present methodology is more 
appealing for experimental investigations, e.g. using planar time-resolved PIV to obtain a three-dimensional mean flow and obtain temporal information at different locations, DNS was employed in the present work to obtain the mean flow and the pointwise data.

The most challenging step of the methodology is the computation of the resolvent modes. For this purpose, a computationally-affordable time-stepping methodology to obtain resolvent modes of complex flows has been introduced, validated and compared to previous existing matrix-free and matrix-forming strategies.

The potential of the present methodology has been demonstrated by application to an unsteady two-dimensional flow around an inclined square cylinder at low Reynolds number. The present approach can predict the fluctuating velocity associated with unsteady separation and the pressure distribution near the square cylinder using just the leading response mode. The temporal evolution of lift and drag coefficients computed from those fields are in good agreement with those obtained via DNS.

\section{REFERENCES}

Barkley, D., Blackburn, H. M. \& Sherwin, S. J. 2008 Direct optimal growth analysis for timesteppers. Int. J. Num. Meth. Fluids 57 (9), 1435-1458.

Blackburn, H. M. \& Sherwin, S. J. 2004 Formulation of a Galerkin spectral element-Fourier method for three-dimensional incompressible flows in cylindrical geometries. J. Comput. Phys. 197, 759-778.

Brunton, S. L., Rowley, C. W. \& Williams, D. R. 2013 Reduced-order unsteady aerodynamic models at low Reynolds numbers. J. Fluid Mech. 724, 203-233.

Gómez, F., Blackburn, H. M., Rudman, M., Sharma, A. S. \& McKeon, B. J. 2016 A reduced-order model of three-dimensional unsteady flow in a cavity based on the resolvent operator. J. Fluid Mech. 798, R2-1-14.

Kurtulus, D. F., Scarano, F. \& David, L. 2007 Unsteady aerodynamic forces estimation on a square cylinder by TR-PIV. Exp. Fluids 42 (2), 185-196.

Lu, L. \& PApadakis, G. 2014 An iterative method for the computation of the response of linearised Navier-Stokes equations to harmonic forcing and application to forced cylinder wakes. Int. J. Num. Meth. Fluids 74 (11), 794-817.

Luhar, M., Sharma, A. S. \& McKeon, B. J. 2014 Opposition control within the resolvent analysis framework. J. Fluid Mech. 749, 597-626.

McKeon, B. J. \& Sharma, A. S. 2010 A critical layer framework for turbulent pipe flow. J. Fluid Mech. 658, 336-382.

Mohrref, R., Sharma, A. S., Tropp, J.A. \& McKeon, B. J. 2013 Model-based scaling of the streamwise energy density in high-Reynolds-number turbulent channels. J. Fluid Mech. 734, 275-316.

Monokrousos, A., Åkervik, E., Brandt, L. \& Henningson, D. S. 2010 Global threedimensional optimal disturbances in the Blasius boundary-layer flow using time-steppers. J. Fluid Mech. 650, 181-214.

Noack, B. R., Afanasiev, K., Morzyński, M., Tadmor, G. \& Thiele, F. 2003 A hierarchy of low-dimensional models for the transient and post-transient cylinder wake. J. Fluid Mech. 497, 335-363.

van Oudheusden, B. W. 2013 PIV-based pressure measurement. Meas. Sci. Tech. 24 (3), 032001.

Sohankar, A., Norberg, C. \& Davidson, L. 1998 Low-Reynolds-number flow around a square cylinder at incidence: study of blockage, onset of vortex shedding and outlet boundary condition. Int. J. Num. Meth. Fluids 26 (1), 39-56.

Spalart, P. R., Jou, W. H., Strelets, M., Allmaras, S. R. et al. 1997 Comments on the feasibility of LES for wings, and on a hybrid RANS/LES approach. Advances in DNS/LES 1, 4-8.

Yoon, D.-H., YANG, K.-S. \& CHOI, C.-B. 2010 Flow past a square cylinder with an angle of incidence. Phys. Fluids 22 (4), 043603. 